\title{
The Use of Probabilistic Neural Network and UV Reflectance Spectroscopy as an Objective Cultured Pearl Quality Grading Method
}

Snezana Agatonovic-Kustrin* and David W Morton

School of Pharmacy and Applied Science, La Trobe Institute of Molecular Sciences, La Trobe University, Australia

\begin{abstract}
Pearl quality and value are determined as a combination of different features, with mollusk species, nacre thickness, luster, surface, shape, color and pearl size, being the most important. A pearl grader has to quantify visual observations and to assign a grading level to a pearl. The aim of this work was to reduce subjectivity in the assessment of some aspects of pearl quality by using artificial neural networks to predict pearl quality parameters from UV reflectance spectra. Given the good predictability of our previous model that used multilayer perceptron ANN modeling of UVVisible spectra to predict the grade of pearls, we wanted to simplify and improve the model by reducing the spectral input to UV only and by using classifier neural network modelling. It is hypothesized that as UV light is of higher energy than visible light, it may penetrate further into the surface of the pearl, and hence the corresponding UV diffuse reflectance spectrum may provide more information that can be used to assess pearl quality.

The developed models were successful in predicting mollusk pearl growing species, pearl and donor color, luster, and surface complexity. The simplified models have been built resulting in more accurate prediction of selected pearl quality parameters when compared with the previous reported model.
\end{abstract}

Keywords: Probabilistic neural network; Diffuse reflectance ultraviolet spectroscopy; Pearl grading; Pearl quality

\section{Introduction}

A pearl grader has to quantify visual observations and to assign a grading level to a pearl. However, there is no international standard method for overall pearl grading [1] and identical pearls may be graded differently by different pearl graders. Usually pearls are classified according to their origin (mussel species) and then graded by assessing the size, nacre thickness, shape, color, luster, and surface (Table 1).

When pearl grading, the appearance of the surface of a pearl is one of the most important characteristics in determining its overall desirability and value. Ideally, the pearl's surface should be smooth, clean and shiny. The presence of imperfections and blemishes on the pearl surface can significantly decrease the value of a pearl, with only $30 \%$ of the cultured pearls harvested categorized as high quality. The surface of a cultured pearl is examined in terms of the number, size, type and location of the imperfection (Table 2).

Blemishes may range from small spots to big chips, or cracks or chalky (calcareous) bumps on the pearl surface [2]. However, in grading South Sea pearls, big ridges forming rings (usually more than

Brilliant, very bright pearl, like a mirror. Light appears to reflect from within the pearl (inner glow)

2 Excellent. A bright pearl but has a slightly blurred reflection

3 High luster. A pearl with minimal inner reelection but blurred

4 Modest luster. A pearl that appears slightly opaque, the reflection is not clear

5 Poor luster. Opaque to the point appearing milky. Commercially not for sale.

Table 1: Lustre category.

A1 No blemishes or one very small blemish that can be removed by drilling.

B1 One to three very small blemishes in close proximity with the majority of the pearl surface being clear

B2 Three or more blemishes but with at least one clean face visible on the pearl Minor blemishes all over the pearl surface (no clean face) or one to two

C1 large blemishes that affect $70 \%$ of the pearl surface. Wrinkled or scratched pearls fall into this category.

C2 Blemishes on entire surface, spots are calcified

D1 A commercially reject pear. A pear that does not fall into above categories.

Table 2: Surface complexity of the pearls. three rings) in a pearl is categorized as a circled pearl. Small spots (noncalcareous) on the surface of a pearl are usually removed by polishing the pearl after harvesting.

Given the good agreement of the previous study that employed the multilayer perceptron (MLP) artificial neural network (ANN) [3] (one of the most commonly used networks), to correlate diffuse reflectance UV-Visible spectra with pearl quality parameters, we wanted to investigate if reducing the number and type of spectral inputs (to UV only) and using the more specific probabilistic neural network (PNN) for correlation we could further improve model predictability. It is well established that the presence of redundant inputs that are not highly related to the outputs, increases the size of a neural network and may result in the network taking longer to train and may provide misleading results. It is hypothesized that as UV light is of higher energy than visible light, it may penetrate further into the surface of the pearl, and hence the corresponding UV diffuse reflectance spectrum may provide more information that can be used to assess pearl quality. A PNN is predominantly a classifier that combines attributes of statistical pattern recognition and feed forward artificial neural networks [4]. In the past decade the ANN modeling technique has found applications for recognition and classification of spectra from a variety of spectroscopic methods [5-9]. Different network architectures, including a MLP, radial basis function (RBF), self-organizing map (SOM), and probabilistic neural network (pnn), have been proposed for classification purposes. However, the PNN has proven to provide a better general solution to pattern classification problems by following Bayesian estimation theory, a statistically developed approach $[10,11]$.

*Corresponding author: Snezana Agatonovic-Kustrin, School of Pharmacy and Applied Science, La Trobe Institute of Molecular Sciences, La Trobe University, Edwards Rd, Bendigo, 3550, Australia, Tel: +61-3-5444-7360; Fax: +61-3-54447878; E-mail: s.kustrin@latrobe.edu.au

Received February 16, 2015; Accepted May 07, 2015; Published May 12, 2015

Citation: Kustrin SA, Morton DW (2015) The Use of Probabilistic Neural Network and UV Reflectance Spectroscopy as an Objective Cultured Pearl Quality Grading Method. Mod Chem appl 3: 152. doi:10.4172/2329-6798.1000152

Copyright: () 2015 Kustrin SA, et al. This is an open-access article distributed under the terms of the Creative Commons Attribution License, which permits unrestricted use, distribution, and reproduction in any medium, provided the original author and source are credited. 


\section{Experimental}

\section{Data collection}

This study was carried out on twenty eight of naturally-colored cultured pearls obtained from commercial pearl farms, 11 freshwater pearls from Zhuji (Zhejiang, China), 4 Akoya pearls from Japan, 5 Tahitian pearls from the South pacific and 8 pearls from a farm in Bali, Indonesia. Pearls were graded according to the South Sea Pearl Grading System issued by Atlas Pacific, Ltd. [12] and had not been subjected to any color or luster enhancing treatments (Table 3). Pearls were classified according to mollusk species, pearl quality (shape, color, luster, and surface complexity), and donor color.

\section{Apparatus}

Since pearls are opaque in nature, spectroscopy measurements were performed using diffuse reflectance spectroscopy. The diffuse reflectance spectra were collected using a Cary 50 UV-Vis spectrophotometer (Varian, Inc.) with a remote diffuse reflectance accessory (DRA) probe (Barrelino TM, Harrick Scientific). The scan rate was $9600 \mathrm{~nm} / \mathrm{min}$. The spectra were measured using appropriate baseline correction at $100 \%$ transmittance. Prior to scanning, the white level was calibrated with a wavelength reflectance standard (Labsphere), in which approximately $100 \%$ reflectance across the entire spectrum is designated as a white reference standard. The spectra were acquired at two different locations on each pearl sample in order to assess surface homogeneity, so that spectral data were independent on the shape of the pearl.

Statistica Neural Networks 9.0 (StatSoft, Tulsa, OK, US) was used to model the spectral data (inputs) and correlate it to pearl quality graded values (outputs). The recorded spectra were post-processed to smooth the noise according to the central moving average method by calculating 102 average spectral values, each from twenty consecutive wavelength [13]. These averaged spectral values were used as inputs to the ANN model. From measured spectral data, training (70\% or a 20 pearl data set), testing ( $15 \%$ or a 4 pearl data set) and validation sets (15\% or a 4 pearl data set) were randomly selected before each training run. Spectral data were used as inputs and pearl quality assessment parameters (mussel species, pearl color and shape complexity, donor color, and donor condition) were used as categorical outputs to train, test, and validate 6 different ANNs.

\section{Artificial neural networks (ANNs)}

An artificial neural network is an information processing model that is inspired by the way biological nervous systems process information and learns from examples. Neural networks are composed of a large number of highly interconnected processing elements or artificial neurons that are organized in successive layers. Each neuron that receives information, process the information and produces an output. Neurons that receive information from outside the network (i.e. spectral data) are called input neurons. Neurons that receive information from other neurons are hidden neurons and neurons whose outputs are used as target values (pearls quality descriptors) are output neurons. Connection weights and the number of hidden neurons in an ANN are adjustable parameters that are optimized during the learning phase.

\begin{tabular}{|l|l|l|l|}
\hline Pearl property & Model topology & Pearl property & Model topology \\
\hline Mollusc species & Linear 93-4 & Lustre & PNN 94-20-5 \\
\hline Pearl colour & Linear 99-12 & Surface complexity & PNN 98-20-6 \\
\hline Donor colour & PNN 97-20-4 & & \\
\hline
\end{tabular}

"Number of inputs-hidden neurons-outputs.

Table 3: Developed ANN models.
There are different ways in which information can be processed by a neuron and different ways of connecting the neuron to one another. Different neural network structures can be constructed by using different processing elements and by the specific way in which they are connected.

After running 30 tests, each evaluating 250 different ANN topologies, linear (LNN) and probabilistic neural networks (PNN) were selected due to their superiority in network performance. A general principal is that a simple model should always be chosen in preference to a complex model if the complex model does not fit the data better. In terms of function approximation, LNN model without hidden layers is the simplest. The network simply multiplies the input by the weights and produces the output.

The architecture of PNN is feed forward, but differs in the way that learning occurs. A PNN is a supervised learning algorithm but includes no weights in its hidden layer. Instead the training data set is acting as the weights to the hidden node. Thus, weights are not adjusted at all. Every training case is copied to the hidden layer of the network, which applies a Gaussian function to the data. The output layer represents each of the possible classes for which the input data can be classified. As no training is required, classifying an input vector is fast, as is simply depends on the number of classes and input data that are present.

\section{Results and Discussion}

The UV diffuse reflectance spectrum is a unique property of a pearl and different pearls will generally have different spectra due to differences in nacre composition (Figures 1-3) [3].

All of the investigated pearls show a decrease in diffuse reflectance at around $260 \mathrm{~nm}$ followed by a peak at around $280 \mathrm{~nm}$ and then a decrease at around $340 \mathrm{~nm}$, regardless of their color (Figures 1-3). Seven Hybrid ANN models with different topology were trained, tested and validated to correlate UV spectra with mollusk pearl growing species, pearl and donor color, luster, and surface complexity (Table 3).

Nondestructive methods for determining the parentage of pearls have practical importance since commercial value of pearls depends on the mother mollusk. The model developed to classify mollusk species was a linear network with 93 spectral inputs and 4 different categories as outputs for four different mollusk species (P. maxima, P. margaritifera, $P$. fucata martensi and freshwater mussel (species unknown for the

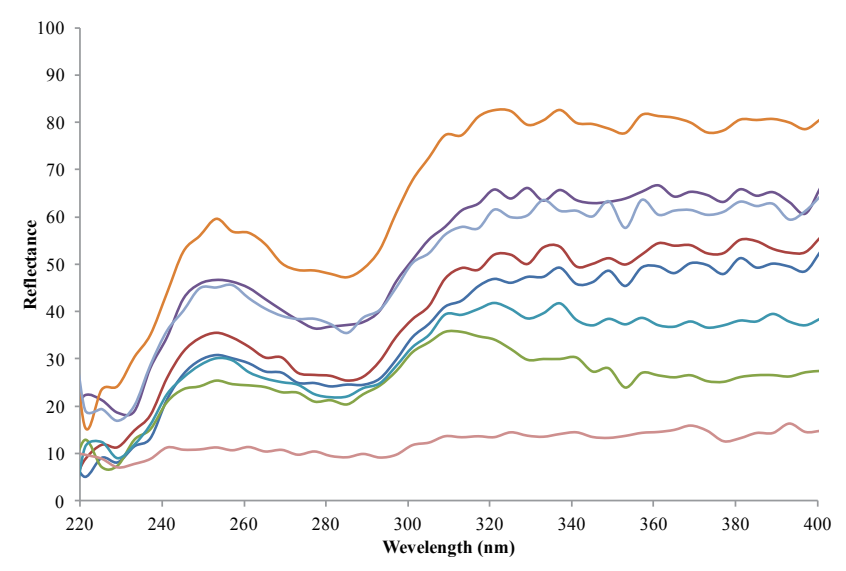

Figure 1: UV Spectral data of eight South sea pearls from Pinctada maxima. 


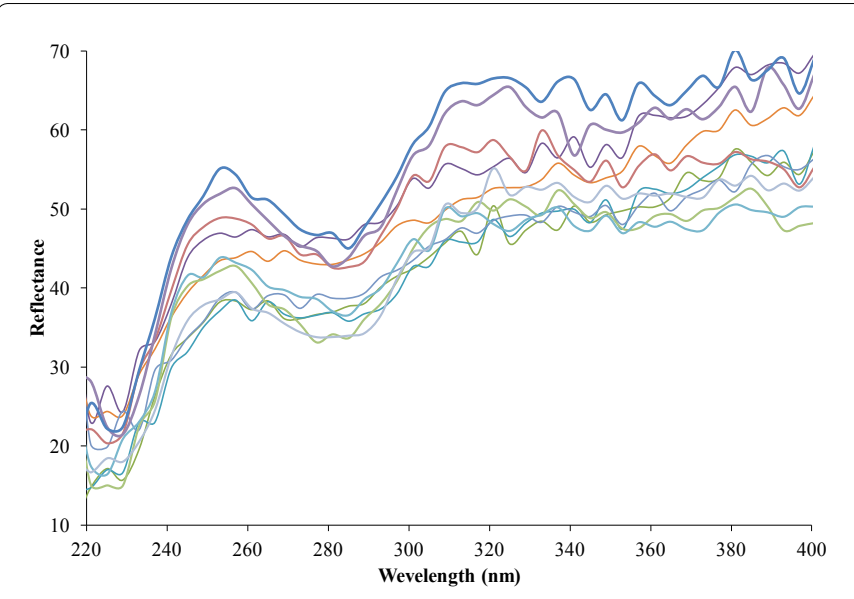

Figure 2: UV spectral data of eleven freshwater pearls from unknown species of freshwater mussels.

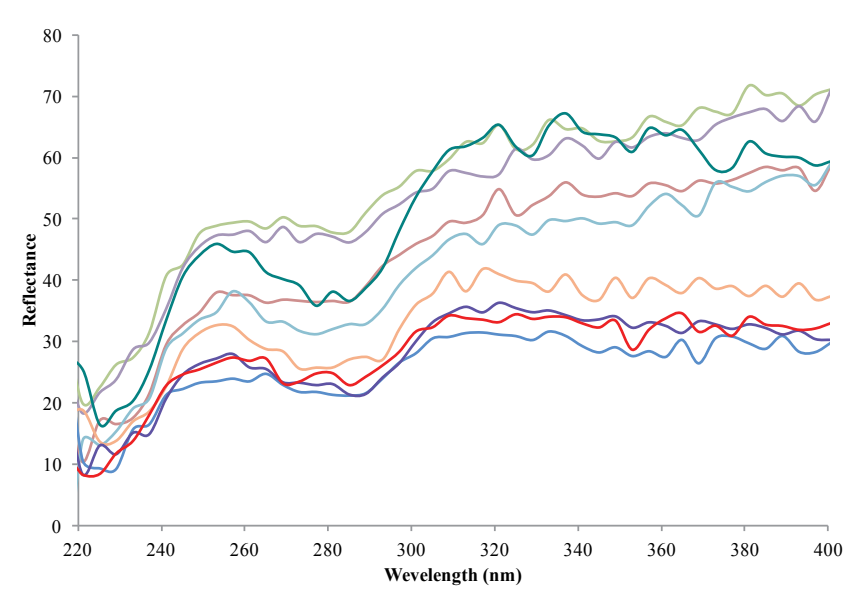

Figure 3: UV spectral data of four Akoya pearls from Pinctada fucata martensi and five Tahitian pearls from Pinctada margaritifera.

freshwater pearls used)). It clearly distinguished between freshwater and saltwater cultured pearls. However for the testing data set, it made mistakes by predicting $P$. margaritifera instead $P$. maxima (sample 2) and $P$. fucata martensi (Japan's Akoya oyster) instead of P. margaritifera (sample 27), while for the validation set it predicted $P$. maxima instead of $P$. margaritifera (sample 26) (Table 4).

The natural colors observed in a pearl are determined by the type of oyster or freshwater mussel that produces the pearl, the water conditions, and the nature of the mantle cells that were used to supply the graft implant. The donor tissue is chosen from oysters with attractive colors in the nacre lining their shells, which gives an indication as to the color of the resulting pearl $[14,15]$. Pearl color is a more subjective indicator of a pearl value, with white South Sea pearls, especially those with a pink overtone, having the highest commercial value [16]. A pearl's color is defined by the nature and the relative proportions of the organic pigments in the nacre [2] (body color), together with reflection and refraction of light [17] (overtone of secondary color).

Using the developed LNN model (99-12), donor color was successfully correlated with the UV spectral data with only one mistake observed (predicting yellow instead unknown donor color (sample
27). However in the case of the pearl color, the developed PNN 97-204 model made a mistake by predicting pink (pearl 2), lavender (pearl 26) and cream with overtone (pearl 27) instead of white (pearl 4) and various colors (pearls 26 and 27) (Table 4).

All natural colors of cultured pearls originate from a mixture of organic pigments, carotenoids or polyenes [18,19]. The P. fucata oyster produces white or cream pearls and doesn't naturally produce black pearls. Cultured pearls from $P$. margaritifera and $P$. maxima are distinguished by their color [1] with $P$. maxima producing pearls with a base color of gold or silver, while $P$. margaritifera produce predominately black color based pearls. P. margaritifera naturally secretes a black pigment, which, depending on the quantity, gives predominantly black pearls, with a basic color ranging from black to gray. P. maxima are a large oyster, also called the gold or silver-lip oyster depending on the color of its shell lip. The oyster from Northern Australia is the silverlip oyster which tends to produce mostly white pearls with a silver overtone. The gold-lip oyster in the sea around Indonesia, Thailand and the Philippines, produces yellow or creamy South Sea pearls [20]. Freshwater cultured pearls occur in four main hues, white, gray, orange and purple. However, due to various combinations of tone and saturation, a broader range of color appearances is observed.

A pearl's color is described by its main body color, overtone or glow, and overtone of secondary color. Usually body colors are enhanced by at least one overtone color. An overtone color is a translucent color that may sometimes appear on a pearl together with its main body color [1] and slightly alters the body color [2]. However, a pearl's overtone color(s) is distinct from its basic color, and this result in pearls in the same color category having very different looks and hues. The phenomenon of overtone of secondary color shows glittering of various colors in a pearl due to the interference and diffraction of light interacting with the specific structure on the pearl's surface $[1,14]$.

Both, yellow and gold cultured pearls exhibit broad absorption from 330 to $460 \mathrm{~nm}$ [21], with two absorption maxima, one between 350 and $365 \mathrm{~nm}$ and the second weaker maximum from 420 to 435 $\mathrm{nm}$. The strength of these absorption maxima increases with increasing saturation of the yellow color. The absence of the UV absorption indicates treated color, regardless of the treatment method used. The relative intensity of the absorption band at $356 \mathrm{~nm}$ is positively correlated to the concentration of the yellow organic pigments present in the nacre [22] (Table 4).

For pearl luster, model (PNN 94-20-5) predicted luster predicted luster 3 , luster 1 and luster 2 instead luster 2, luster 2 and luster 1 for the samples 10,15 and 26. The pearl's surface luster is critical in evaluating pearl quality. It depends upon the reflection and refraction of light from the translucent layers of the nacre (Table 1). The luster of a pearl may be closely related to the homogeneity, light transmittance and quality of the nacre. A pearl's luster is a measure of its brilliance and the reflectivity of a pearl. High-quality pearls are bright and shiny; with high reflectivity (mirror-like reflectivity) while lower-quality pearls have a chalky or dull appearance. The error in the prediction of the surface complexity was minor. However, there was an issue with South Sea pearl sample coded 2, where the model predicted B2 (one to three very small blemishes) instead of C1 (minor blemishes all over the pearl or one or two large blemishes) (Table 2). It is important to note that no predictive model will be $100 \%$ accurate. We also need to recognize that pearls graded by humans are also not $100 \%$ accurately graded. For example, there can be differences in peoples' perception of luster. Given that our developed model is dependent on actual graded pearls, any error made in the grading of these pearls will affect the accuracy of the 
Citation: Kustrin SA, Morton DW (2015) The Use of Probabilistic Neural Network and UV Reflectance Spectroscopy as an Objective Cultured Pearl Quality Grading Method. Mod Chem appl 3: 152. doi:10.4172/2329-6798.1000152

Page 4 of 6

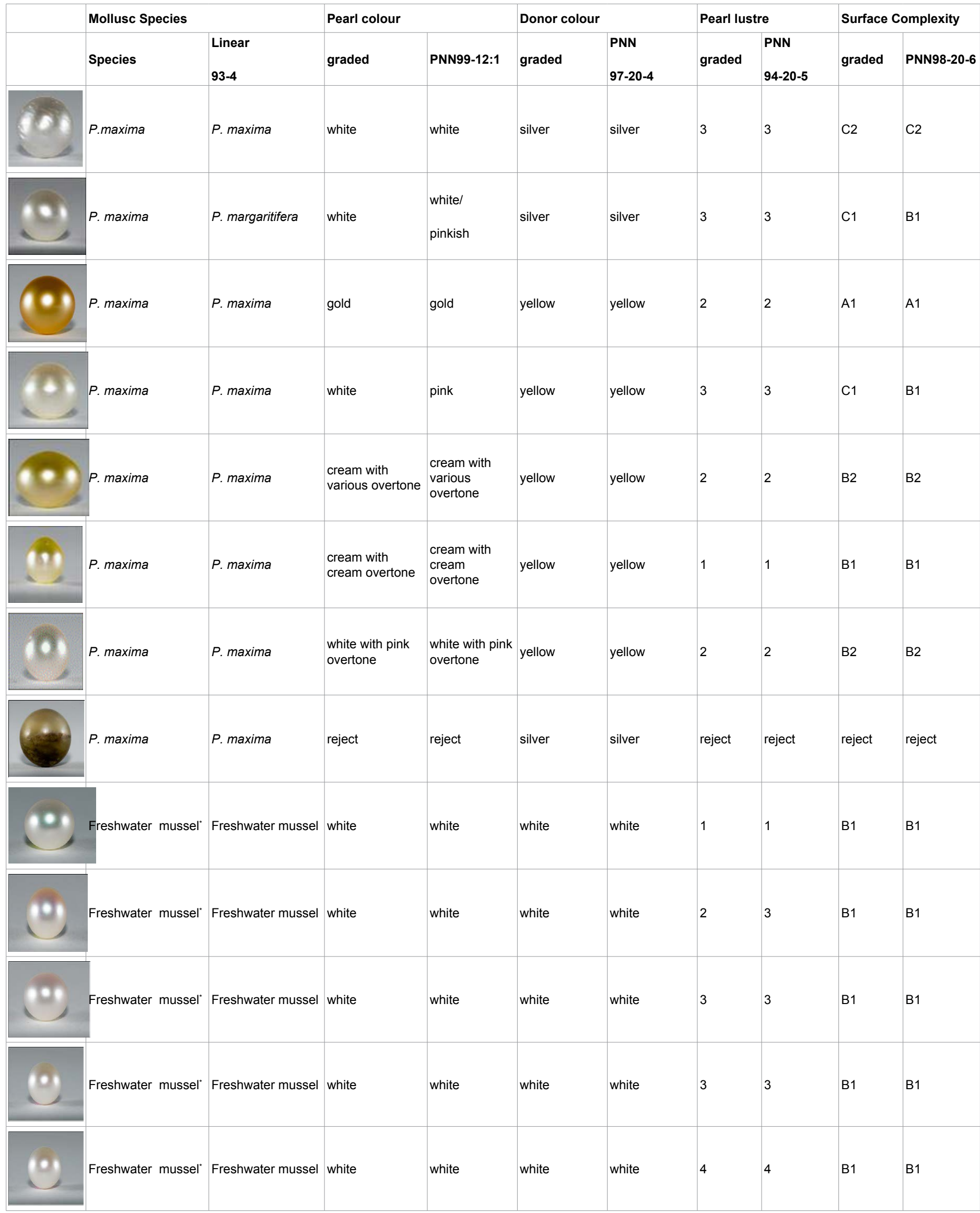


Citation: Kustrin SA, Morton DW (2015) The Use of Probabilistic Neural Network and UV Reflectance Spectroscopy as an Objective Cultured Pearl Quality Grading Method. Mod Chem appl 3: 152. doi:10.4172/2329-6798.1000152

Page 5 of 6

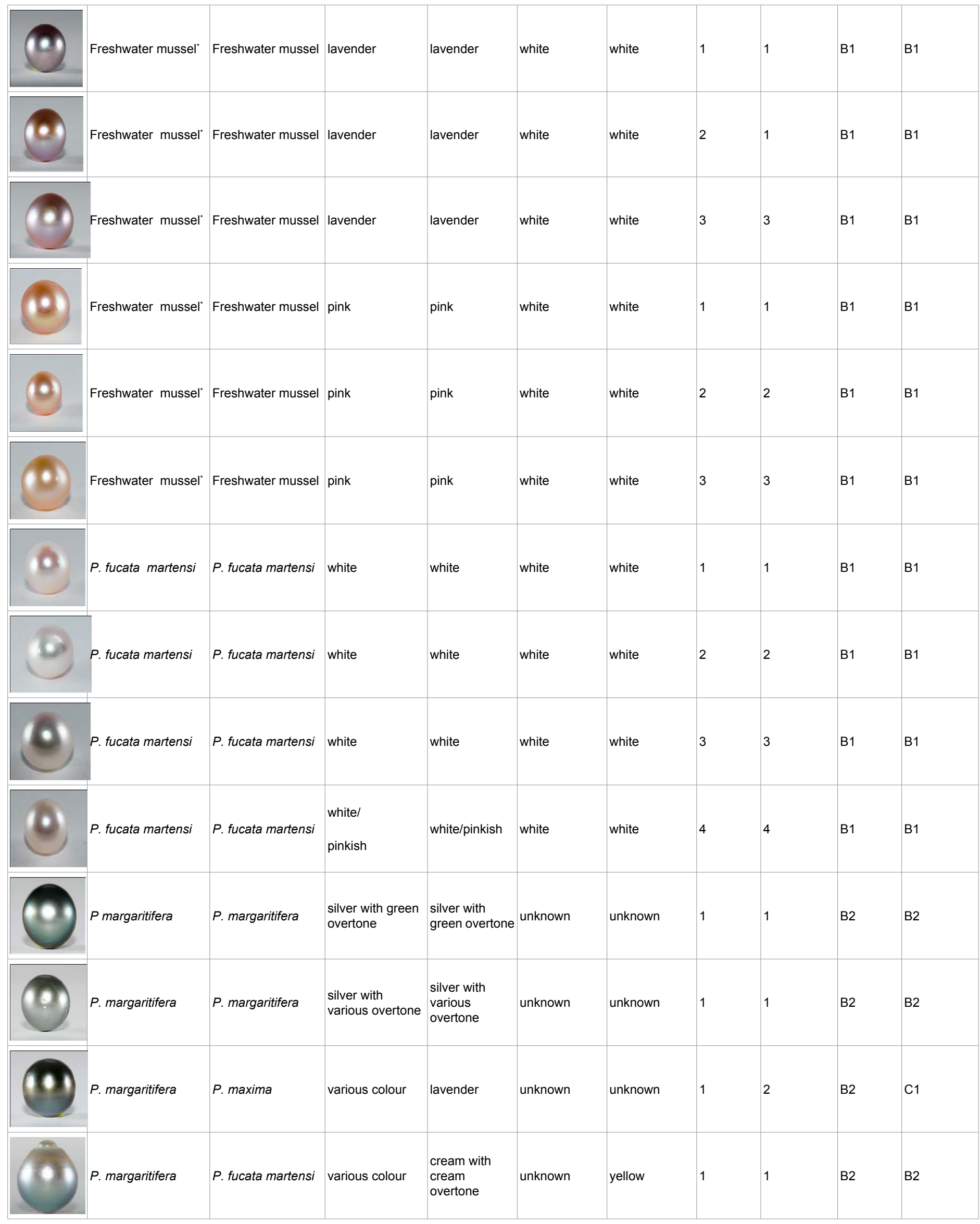


Citation: Kustrin SA, Morton DW (2015) The Use of Probabilistic Neural Network and UV Reflectance Spectroscopy as an Objective Cultured Pearl Quality Grading Method. Mod Chem appl 3: 152. doi:10.4172/2329-6798.1000152

Page 6 of 6

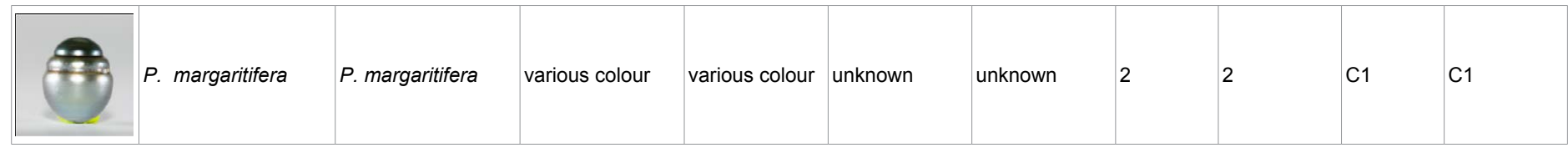

*Species unknown.

Table 4: Mollusc species graded and predicted pearl and donor colour, lustre and surface complexity using the optimised ANN models.

model. It is important to note that the advantage of using a predictive model of this type is that it is not subject to the errors associated with the use of human graders provided an accurate set of graded pearls is used in the modelling process and a predictive model of high accuracy is produced. This approach will minimize the difference between pearls graded using a predictive model and graders.

\section{Conclusions}

Diffuse reflectance UV spectroscopy combined with PNN data modeling, successfully classified and graded 28 different pearls, resulting in more accurate prediction of selected pearl quality parameters (mollusk species, pearl and color donor, luster, and surface complexity) when compared with the previous model [3]. 25 out of 28 mollusk species were correctly classified (90\%) and similarly $90 \%$ of pearl color was correctly predicted with developed models. In the case of donor color only 1 prediction was wrong and for the luster and surface complexity relative error was $7 \%$ (2 out of 28 ). The simplified LNN and PNN models have been developed without any loss of accuracy in prediction. There are still few prediction errors observed, most likely to be attributable to the relatively small number of pearl samples. The proposed method may thus provide non-destructive and objective evaluation of pearl quality within minutes and rapid scanning of large number of pearls using simple and cost effective apparatus.

\section{References}

1. Wegst UGK, Ashby MF (2004) The mechanical efficiency of natural materials. Philosophical Magazine 84: 2167-2181.

2. Olmos P, Diaz JC, Perez JM, Gomez P (1991) A new approach to automatic radiation spectrum analysis. IEEE Transactions on Nuclear Science 38: 971975.

3. Agatonovic-Kustrin S, Morton DW (2012) The use of UV-visible reflectance spectroscopy as an objective tool to evaluate pearl quality. Mar Drugs 10: 1459-1475.

4. Ancona F, Colla A, Rovetta S, Zunino R (1997) Implementing probabilistic neural networks. Neural Comput \& Applic 7: 37-51.

5. Taylor JJU, Strack E (2008) Pearl production, Elsevier, Amsterdam.

6. Agatonovic-Kustrin S, Tucker IG, Schmierer D (1999) Solid state assay of ranitidine $\mathrm{HCl}$ as a bulk drug and as active ingredient in tablets using DRIFT spectroscopy with artificial neural networks. Pharm Res 16: 1477-1482.

7. Sarikaya M, Aksay IA (1995) Biomimetics: Design and Processing of Materials. AIP Press, Woodbury, NY, USA.

8. Amendolia SR, Doppiu A, Ganadu ML, Lubinu G (1998) Classification and quantitation of $1 \mathrm{H}$ NMR spectra of alditols binary mixtures using artificial neural networks. Anal Chem 70: 1249-1254.

9. Bos M, Weber HT (1991) Comparison of the training of neural networks for quantitative x-ray fluorescence spectrometry by a genetic algorithm and backward error propagation. Anal Chim Acta 247: 97-105.

10. Romero RD, Touretzky DS, Thibadeau RH (1997) Optical Chinese character recognition using probabilistic neural networks. Pattern Recognition 30: 12791292.

11. Specht DF (1990) Probabilistic Neural Networks. Neural Networks 3: 109-118.

12. Shadmehr R, Angell D, Chou PB, Oehrlein GS, Jaffe RS (1992) Principal component analysis of optical emission spectroscopy and mass spectrometry: Application to reactive ion etch process parameter estimation using neural networks. J Electrochem Soc 139: 907-914.
13. Milosevic M, Berets SL (2006) Accessories and Sample Handling for MidInfrared Diffuse Reflection Spectroscopy. In: Chalmers JM, Griffiths P (eds.) Handbook of Vibrational Spectroscopy. John Wiley \& Sons, Ltd, USA.

14. Acosta-Salmón H, Martinez-Fernández E, Southgate PC (2004) A new approach to pearl oyster broodstock selection: can saibo donors be used as future broodstock? Aquaculture 231: 205-214.

15. Mamangkey NG, Agatonovic S, Southgate PC (2010) Assessing pearl quality using reflectance UV-Vis spectroscopy: does the same donor produce consistent pearl quality? Mar Drugs 8: 2517-2525.

16. Gilbert PU, Metzler RA, Zhou D, Scholl A, Doran A, et al. (2008) Gradual ordering in red abalone nacre. J Am Chem Soc 130: 17519-17527.

17. Raman CV, Krishnamurti D (1954) On the chromatic diffusion halo and other optical effects exhibited by pearls. Proceedings of the Indian Academy of Sciences 39A: 265-271.

18. de Oliveira VE, Castro HV, Edwards HGM, de Oliveira LFC (2009) Carotenes and carotenoids in natural biological samples: a Raman spectroscopic analysis. Journal of Raman Spectroscopy 41: 642-650.

19. Karampelas S (2007) Pigments in natural-color corals. Gems \& Gemmology 43: 95-96.

20. Edge R, McGarvey DJ, Truscott TG (1997) The carotenoids as anti-oxidants--a review. J Photochem Photobiol B 41: 189-200.

21. Elen $S$ (2001) Spectral reflectance and fluorescence characteristics of naturalcolor and heat-treated "golden" south sea cultured pearls. Gems \& Gemology 37: 114-123.

22. Li-jian Q, Yi-lan H, Zeng CG (2008) Colouration Attributes and UV-NIS Reflection Spectra of Various Golden Seawater Cultured Pearls. Gems \& Gemnology 10: 1-8. 